\section{Mut zur Geduld beim Neuroblastom}

\author{
Für die Prognose beim Neuroblastom hat die Amplifikation des Onkogens \\ MYCN große Bedeutung. Liegt keine MYCN-Amplifikation vor, kann \\ möglicherweise bei disseminierten Neuroblastomen im ersten Lebensjahr \\ auf eine Chemotherapie verzichtet werden.
}

B eim Neuroblastom besteht eine starke Korrelation zwischen MYCN-Amplifikation und fortgeschrittenem Stadium der Erkrankung sowie rascher Tumorprogression. Wissenschaflter aus Italien begannen deshalb zwei prospektive Studien, in denen 170 Patienten mit disseminiertem Neuroblastom ohne MYCNAmplifikation nur dann eine Chemotherapie erhielten, wenn lebens- oder organbedrohende Symptome oder Metastasen in Skelett, Lunge oder ZNS auftraten. Operiert wurden die Patenten nur, wenn kein besonderes Operationsrisiko vorlag.

Die Studie 99.2 umfasste 125 Patienten im Stadium 4S, also Kinder im ersten Lebensjahr mit lokalem Primärtumor (Stadium 1, 2A oder 2B) und Absiedlungen, die auf Haut, Leber und Knochenmark beschränkt waren, sowie solche im Stadium 4 mit einem Primärtumor, der die Mittellinie überschritten hatte oder mit einem positiven Befund in der Skelettszintigrafie, die bei Symptomfreiheit nur beobachtet wurden.

In der Studie 99.3 wurden 45 Kinder mit Metastasen von Skelett, Lunge oder ZNS mit bis zu vier Chemotherapiezyklen (Carboplatin plus Etoposid) behandelt. Bei Versagen folgten bis zu vier Zyklen mit Cyclophosphamid, Doxorubicin und Vincristin. Diese Chemotherapie stand auch für Kinder der Studie 99.2 zur Verfügung, wenn sie starke Symptome entwickelten.

Das Zwei-Jahres-Überleben lag in der Studie 99.2 bei $97,6 \%$. Dabei fanden die Autoren keine Unterschiede zwischen Kindern mit und ohne Symptome (97,7 vs. 97,3\%) oder mit einem nicht resektablen und einem resektablen Tumor (96,8 vs. $100 \%$ ). Auch die Kinder in Studie 99.3 hatten mit einer Zwei-JahresÜberlebensrate von 95,6\% eine gute Prognose. Kein Patient verstarb aufgrund von Komplikationen im Gefolge von Chirurgie oder Chemotherapie.

Liegt keine MYCN-Amplifikation vor, haben Kinder mit disseminiertem Neuro- blastom auch bei zurückhaltender oder gar keiner Therapie eine sehr gute Prognose. Deshalb empfehlen die Autoren in einem solchen Falle und bei Fehlen von Symptomen und radiologischen Auffälligkeiten eine reine Beobachtung.

Kommentar: Der prognostisch wichtigste und möglicherweise übergeordnete genetische Marker der Neuroblasten ist der Amplifikationsstatus des MYCNGens. Patienten mit normaler Kopienzahl des MYCN-Gens haben eine wesentlich günstigere Prognose als Patienten, bei denen dieses Gen amplifiziert ist. In der derzeit aktiven deutschen NeuroblastomStudie erhalten Patienten mit normalem MYCN-Status ihrer Neuroblastome eine wesentlich kürzere und weniger intensive Polychemotherapie als Patienten mit Amplifikation des MYCN-Gens.

In den vorliegenden beiden Studien 99.2 und 99.3 beschreiben de Bernardi und Mitarbeiter erstmals eine prospektive Therapiestudie, bei der Säuglinge mit einem disseminierten Neuroblastom ohne MYCN-Amplifikation nur dann eine Chemotherapie erhielten, wenn lebensbedrohliche Komplikationen auftraten oder durch Röntgen bzw. Com- putertomografie entdeckte Skelettmetastasen nachweisbar waren. Eine Operation erfolgte in dieser Studie nur dann, wenn aufgrund von Größe und Lokalisation des Tumors keine besonderen Operationsrisiken bestanden.

Die vorliegende Studie beweist erstmals und eindrucksvoll, dass auch in einer prospektiven Therapiestudie Säuglinge ohne MYCN-Amplifikation ein exzellentes Überleben mit nur minimaler Therapie und vor allem auch ohne eine spezifische Polychemotherapie aufweisen. Die Autoren schließen daraus, dass asymptomatische Säuglinge, deren Neuroblastome keine MYCN-Amplifikation aufweisen, mit einem nicht resezierbaren Primärtumor und/oder pathologischer Skelettszintigrafie bei normalem Skelettröntgen bzw. -CT zukünftig keiner spezifischen Therapie bedürfen, sondern dass es gerechtfertigt ist, sie nur zu beobachten und den bei fast allen Säuglingen mit Neuroblastom ohne MYCN-Amplifikation günstigen Spontanverlauf abzuwarten. Allerdings war dieses - onkologisch ungewöhnliche - Vorgehen nicht allen an der Studie beteiligten pädiatrischen Onkologen vermittelbar: 30 asymptomatische Säuglinge wurden entgegen den Richtlinien des Protokolls dennoch mit einer Chemotherapie behandelt.

Prof. Dr. Jörg Ritter

Bernardi B de et al. Excellent outcome with reduced treatment for infants with disseminated neuroblastoma without MYCN gene amplification. J Clin Oncol 2009; 27: $1034-40$.

\section{HPV-Impfschutz auch nach sechseinhalb Jahren belegt}

Eine kanadische Studie ermittelte Langzeitdaten zur Wirksamkeit und Immunogenität des bivalenten HPV-Impfstoffes. 1.113 Frauen im Alter zwischen 15 und 25 Jahren mit unauffälliger Zytologie nahmen daran teil. Alle waren seronegativ für HPV 16 und 18. Bei 560 Teilnehmerinnen wurde eine Impfung mit der bivalenten Vakzine gegen HPV 16 und 18 durchgeführt, die anderen erhielten ein Placebopräparat.

Mittlerweile liegen Daten für die Zeitspanne von bis zu 6,4 Jahren nach der Impfung vor. Die Impfeffektivität gegen Infektionen mit den Serotypen 16 und 18 lag bei 95,3\%. Eine zwölfmonatige Persistenz der Viren konnte zu 100\% verhindert werden. Für zervikale intraepitheliale Neoplasien vom Grad 2 oder höher (CIN2+), die durch die Serotypen 16 und 18 ausgelöst worden waren, betrug die Impfeffektivität ebenfalls 100\%. Die Antikörperkonzentrationen blieben mindestens um das Zwölffache gegenüber dem Titer bei einer natürlichen HPV-Infektion erhöht.

Romanowski B et al. Sustained efficacy and immunogenicity of the human papillomavirus (HPV)-16/18 ASo4-adjuvanted vaccine: analysis of a randomised placebocontrolled trial up to 6.4 years. Lancet 2009; $374: 1975-85$ 